\title{
Achados histopatológicos em úteros e ovários de cadelas submetidas à castração eletiva pelas técnicas de ovariectomia ou ovariohisterectomia
}

\author{
[Histopathological findings in the uterus and ovaries of bitches undergoing elective gonadectomy \\ via ovariectomy or ovaryhisterectomy techniques]
}

\section{"Artigo Científico/Scientific Article"}

\author{
Karine Silva Camargo ${ }^{1 *}$, Grazielle Anahy de Sousa Aleixo ${ }^{1}$, Mauro de Araújo Penaforte Júnior ${ }^{1}$, \\ Gabriela Ratis Galeas ${ }^{1}$, Sabrina Cândido Trajano ${ }^{1}$, Kleyton Domingos de Melo ${ }^{1}$, \\ Maria Sheila da Silva Ferreira ${ }^{1}$, Lilian Sabrina Silvestre de Andrade ${ }^{1}$, Levi Auto Lopes ${ }^{2}$
}

\begin{abstract}
${ }^{1}$ Departamento de Medicina Veterinária, Universidade Federal Rural de Pernambuco (UFRPE), Recife-PE, Brasil. ${ }^{2}$ Departamento de Zootecnia, Universidade Federal Rural de Pernambuco (UFRPE), Recife-PE, Brasil.

*Autor para correspondência/Corresponding author: E-mail: karinecamargo01@gmail.com
\end{abstract}

\begin{abstract}
Resumo
Objetivou-se avaliar os achados histopatológicos dos ovários e úteros de cadelas submetidas à ovariectomia (OVE) ou ovariohisterectomia $(\mathrm{OVH})$ visando identificar afecções ovarianas e/ou uterinas não diagnosticadas durante exame clínico, laboratorial (hemograma e bioquímico) e ultrassonográfico pré-operatório ou macroscopicamente no transcirúrgico. Foram selecionadas 20 cadelas clinicamente saudáveis e não prenhes provenientes da casuística do Hospital Veterinário da Universidade Federal Rural de Pernambuco, que foram divididas em Grupo OVH (10 animais) e Grupo OVE (10 animais). Foram coletados os ovários e úteros obtidos durante o procedimento de OVH, e ovários e fragmentos de $0,5 \mathrm{~cm}$ do corno uterino direito e esquerdo foram coletados durante a OVE, sendo estes encaminhados para avaliação histopatológica. Os resultados demonstraram a presença de hiperplasia endometrial cística em $80 \%$ dos animais submetidos à OVH e em 40\% dos animais do grupo OVE. No grupo OVE, foi identificada a presença de hiperplasia adenomatosa de rete ovarii em uma paciente (10\%) e adenoma de rete ovarii em outra $(10 \%)$, alterações estas consideradas raras na medicina veterinária. Conclui-se que o exame histopatológico é fundamental no diagnóstico de alterações ovarianas e/ou uterinas não detectadas em exame clínico, ultrassonográfico, laboratorial (hemograma e bioquímico) e macroscópico de cadelas submetidas à castração eletiva pelas técnicas de OVH e OVE.
\end{abstract}

Palavras-chave: alterações; cirurgia; contraceptivo; rete ovarii.

\begin{abstract}
The objective of this study was to evaluate the histopathological findings in the ovaries and uterus of bitches submitted to ovariectomy (OVE) or ovariohysterectomy (OVH) to identify undiagnosed ovarian and/or uterine conditions during clinical, laboratory (blood count and biochemist), and ultrasound examination preoperatively or macroscopically during the surgery. Twenty clinically healthy and non-pregnant bitches from the Veterinary Hospital of the Federal Rural University of Pernambuco were divided into OVH Group (10 animals) and OVE Group (10 animals). The ovaries and uterus obtained during the $\mathrm{OVH}$ procedure, and the ovaries and two $0.5 \mathrm{~cm}$ fragments of the right and left uterine horns collected during OVE were submitted for histopathological evaluation. The results demonstrated the presence of cystic endometrial hyperplasia in $80 \%$ of the animals submitted to $\mathrm{OVH}$ and in $40 \%$ of the OVE group. In the OVE group, the presence of adenomatous hyperplasia of rete ovarii in one patient (10\%) and rete ovarii adenoma in another (10\%) were identified, which are considered rare conditions in veterinary medicine. It is concluded that the histopathological examination is fundamental for the diagnosis of ovarian and/or uterine alterations that were not observed in the clinical, ultrasonographic, laboratory (blood count and biochemist), and macroscopic examination of bitches submitted to elective castration by OVH and OVE techniques.
\end{abstract}

Keywords: adenoma; surgery; contraceptive; rete ovarii. 


\section{Introdução}

Atualmente, a castração de caninos e felinos é um procedimento cirúrgico eletivo rotineiro na medicina veterinária, devido à necessidade do controle reprodutivo e populacional, e das possíveis afecções associadas à não realização da mesma. Dentre as técnicas de castração existentes a ovariohisterectomia $(\mathrm{OVH})$ e a ovariectomia (OVE) são os procedimentos cirúrgicos mais realizados em estabelecimentos privados e instituições de ensino (Santos et al., 2009).

Alguns autores apontam a OVE como a melhor técnica de castração de fêmeas devido ao fato de serem realizadas incisões menores em comparação a $\mathrm{OVH}$, diminuindo assim o trauma abdominal, o tempo cirúrgico e o risco anestésico (Okkens, 2002; Shariati, 2014). Entretanto, a OVE não é indicada nos casos de enfermidades que acometem o útero, piometra, hidrometra, mucometra e torção uterina (Ehrhardt, 2012), sendo nestes casos a OVH indicada como tratamento de eleição. Existem alterações que podem, em algumas situações, não apresentar sinais evidentes ou serem discretas, como em casos de mucometra e neoplasias em estágios inicias (Nascimento e Santos, 2011; Aguirra et al., 2015), evidenciando a importância da realização de exames físicos e complementares, como hemograma, ultrassonografia e biopsia para realização de pré-diagnóstico e implementação da técnica cirúrgica adequada como forma de tratamento.

O exame histopatológico é indicado para avaliar microscopicamente os tecidos para a detecção de possíveis lesões existentes (Monteiro et al., 2009) e tem sido fundamental no diagnóstico de distúrbios uterinos, como nos casos de hiperplasia endometrial cística (HEC) (Dow, 1959; De Bosschere et al., 2003; Carreira e Pires, 2005). As neoplasias também são alterações que podem ser observadas e diagnosticadas no exame histopatológico (Jones et al., 2000; Daleck et al., 2009). Embora sejam raros no útero, correspondendo a $0,4 \%$ de todos os tumores em cadelas (Araujo et al., 2015), o leiomioma, fibroma, fibroleiomioma e leiomiossarcoma são os mais comuns (Maclachlan e Kennedy, 2002).

Diante do exposto e da hipótese de que afecções nos tecidos ovarianos e uterinos podem não ser detectadas nos exames complementares pré-cirúrgicos, objetivou-se avaliar alterações histopatológicas de úteros e ovários de cadelas submetidas à ovariohisterectomia e ovariectomia.

\section{Material e Métodos}

Foram selecionadas 20 cadelas provenientes da rotina do Hospital Veterinário (HV) da UFRPE, com idade entre 1 e 6 anos, com peso de 7 a 13 quilos e sem distinção de raça.

Os animais foram divididos aleatoriamente em dois grupos: Grupo OVH $(n=10)$ onde foi realizado o procedimento de ovariohisterectomia e Grupo OVE $(n=10)$ no qual foi realizada ovariectomia.

Para avaliação do quadro clínico e do risco cirúrgico das pacientes, foram realizados os seguintes exames pré-operatórios: hemograma, bioquímica hepática (dosagem de ALT, AST e FA) e renal (dosagem de ureia e creatinina) e ultrassonografia de abdômen total. Somente os animais sem alterações nos exames realizados e que não estavam prenhes foram considerados aptos para participarem da pesquisa e foram submetidos aos procedimentos cirúrgicos.

Todas as cirurgias foram realizadas pelo mesmo cirurgião e com a ajuda de um auxiliar, utilizando nos procedimentos de OVH e OVE as técnicas padrões descritas na literatura (Howe, 2006; Macphail, 2014).

Para análise histopatológica, foram coletados os úteros removidos durante o procedimento de $\mathrm{OVH}$, e dois fragmentos de 0,5 $\mathrm{cm}$ medidos com paquímetro (um do corno uterino direito e outro do esquerdo) durante a OVE, os quais foram coletados imediatamente abaixo da ligadura do pedículo ovariano, além dos ovários. O material foi acondicionado em recipiente de polipropileno, com solução de formalina neutra a $10 \%$, devidamente identificado e encaminhado para processamento histopatológico, onde foram submetidas à desidratação em amostras de álcool decrescente $(70 \%, 80 \%, 90 \%$, álcool absoluto 1,2 e 3), diafanização (xilol absoluto 1 e 2), embebição em parafina 1 e 2 e inclusão em parafina histológica derretida a $60^{\circ} \mathrm{C}$. Em seguida, foram submetidas à microtomia para a secção de cortes histológicos de cinco micrômetros de espessura que foram depositados em lâminas histológicas e coradas por hematoxilina-eosina (Luna, 1968) e analisadas em microscópio óptico.

Para análise dos dados adotou-se a metodologia de análise descritiva em percentual da frequência das alterações histológicas dos dados obtidos (Pimentel, 1985). 


\section{Resultados e Discussão}

$\mathrm{Na}$ análise histopatológica do útero das cadelas submetidas à $\mathrm{OVH}$, observou-se $\mathrm{o}$ predomínio do complexo hiperplasia endometrial cística $(80 \%$ - 8/10) e apenas $20 \%(2 / 10)$ das cadelas avaliadas não apresentaram alterações histopatológicas.

Dos animais diagnosticados com hiperplasia endometrial cística três cadelas foram classificadas como HEC Tipo I, três como Tipo II e duas como Tipo III (Figuras 1, 2 e 3), de acordo com a classificação proposta por Dow (1959).

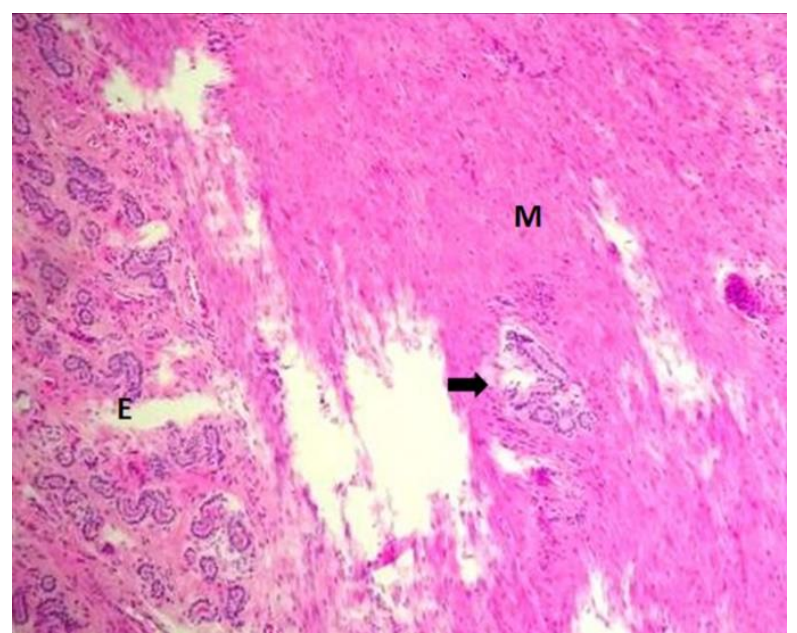

Figura 1. Hiperplasia de glândulas endometriais difusa moderada com reação pseudoplacentacional difusa moderada e adenomiose focal discreta (complexo hiperplasia endometrial cística tipo I seta); M - região medular. Coloração de hematoxilina e eosina (HE), objetiva de $20 x$.

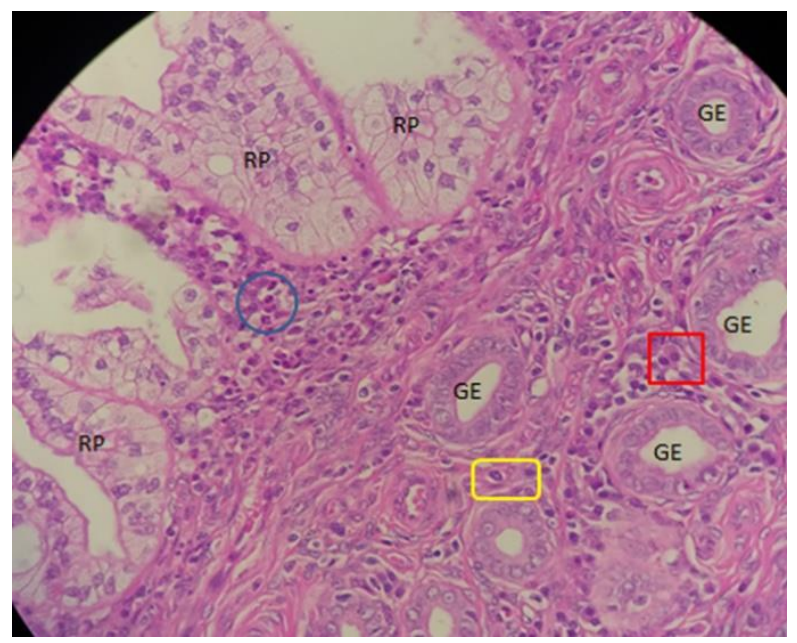

Figura 2. Endométrio com ectasia de glândulas endometriais (GE) difusa acentuada e infiltrado difuso discreto de linfócitos (retângulo amarelo), plasmócitos (quadrado vermelho) e neutrófilos (círculo azul). Complexo hiperplasia endometrial cística tipo III, com reação pseudoplacentacional (RP). Coloração de hematoxilina e eosina (HE), objetiva de 40x.

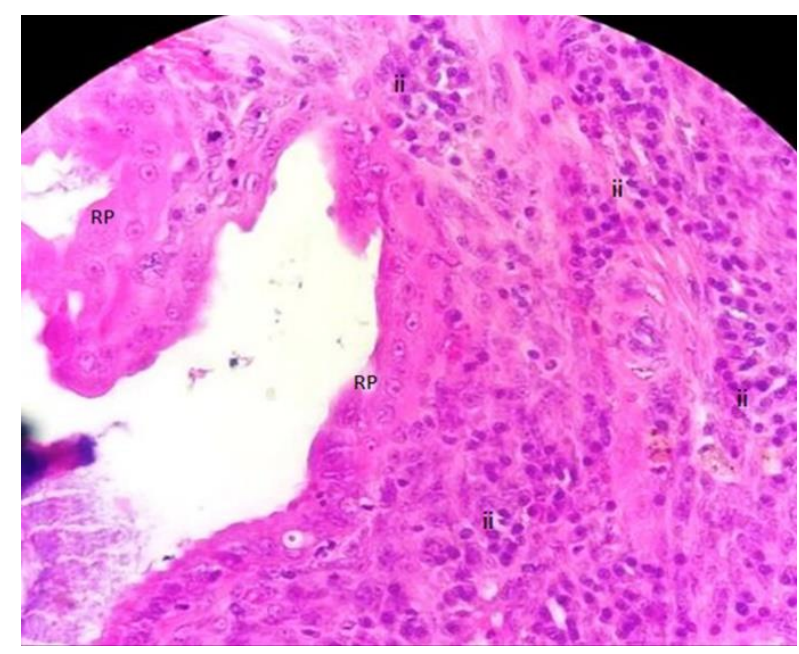

Figura 3. Epitélio do endometrial luminal e das glândulas com células acentuadamente volumosas, citoplasma moderadamente vacuolizado e fracamente eosinofílico, reação pseudoplacentacional (RP) e lâmina própria com infiltrado inflamatório (ii) de linfócitos. HEC-tipo III.

A HEC é uma alteração endometrial do útero de cadelas que se desenvolve durante o diestro e está correlacionada com os altos níveis de estrógeno e exposição prolongada de progesterona e/ou quando esta é administrada de forma exógena (Fossum, 2005). Geralmente este complexo é assintomático e o diagnóstico feito tardiamente (Fossum, 2005; Veiga et al., 2017) o que justifica as cadelas do presente estudo apresentarem a doença dos Tipos I, II e III sem que tivessem apresentado alterações clínicas previamente à realização da cirurgia.

Segundo Dow (1959) a HEC tipo I é caracterizada por não apresentar nenhuma reação inflamatória, mas uma proliferação das glândulas endometriais, podendo elas estar ou não císticas; a do Tipo II está associada à discreta infiltração inflamatória (com neutrófilos e macrófagos); a Tipo III possui uma intensidade variável, associada à infiltração inflamatória, com linfoplasmocitária e neutrofílica e com comprometimentos mais severos, enquanto a de grau IV está associada a uma fibrose do miométrio. A HEC tipo IV não foi diagnosticada no presente estudo, porém as do tipo I, II e III puderam ser determinadas conforme descrito acima, e seus achados, similares aos descritos pelo autor.

A análise histopatológica do grupo submetido à OVE demonstrou que $10 \%$ dos ovários analisados (1/10) apresentaram adenoma de rete ovarii (Figura 4) e 10\% (1/10) apresentaram hiperplasia adenomatosa de rete ovarii (Figura 5). $\mathrm{O}$ rete ovarii é um local incomum para qualquer lesão e adenomas de rete são achados ocasionais 
(Jiang et al., 2004). Segundo Kennedy et al. (1998), de acordo com a classificação histológica de tumores em animais domésticos, hiperplasia adenomatosa de rete ovarii é classificada como uma lesão tumoral, sendo considerada uma lesão rara, observada principalmente em humanos (McEntee, 1990; Nogales et al., 1997) e em cães (McEntee, 1990; Jiang et al., 2004). Levando em consideração o grande número de fêmeas que são castradas na clínica de pequenos animais, se todos os ovários e úteros removidos fossem submetidos à análise histológica, haveria uma grande contribuição para o estudo de prevalência de doenças ovarianas e uterinas, inclusive de afeções raras, como é o caso do adenoma e hiperplasia adenomatosa de rete ovarii.

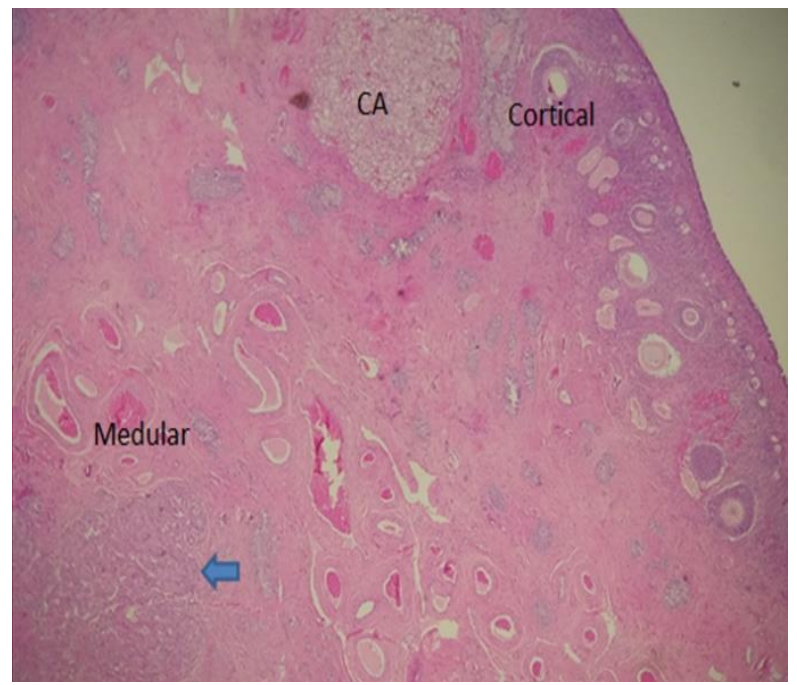

Figura 4. Região medular com hiperplasia adenomatosa de rete ovarii (seta) em ovário de cadela submetida à ovariectomia.; CA- corpus albicans - objetiva 40x.

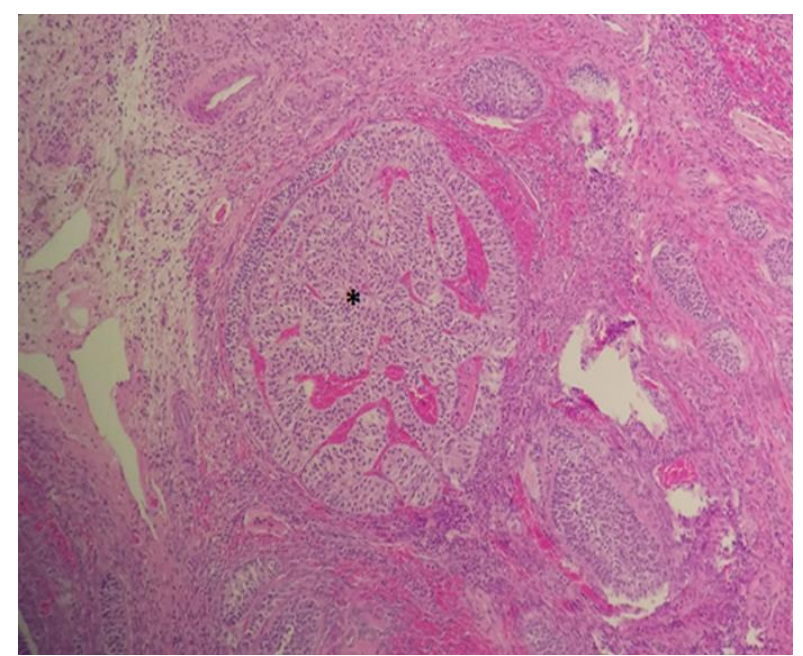

Figura 5. Adenoma de rete ovarii (asterisco). Coloração de hematoxilina e eosina (HE), objetiva de 20x.

Dos fragmentos dos úteros deste mesmo grupo, $60 \%$ (6/10) não apresentaram alteração e
40\% apresentaram HEC; destes, 20\% (2/10) corresponderam ao Tipo I e 20\% (2/10) ao Tipo II.

Apesar dos exames histopatológicos apresentarem alterações de HEC em ambos os grupos, vale salientar que somente os animais clinicamente saudáveis foram considerados aptos para participarem da pesquisa e foram submetidos aos procedimentos cirúrgicos. Ainda assim, alterações uterinas macroscópicas foram observadas no transcirúrgico em 30\% (6/20) dos animais avaliados, embora as pacientes não tivessem apresentado alterações indicativas nos exames ultrassonográficos, hemograma e bioquímico.

Apesar da importância da realização dos exames de avaliação pré-operatória, a experiência do cirurgião deve prevalecer na decisão sobre a necessidade de retirar o útero no momento transcirúrgico. Macroscopicamente, os úteros apresentavam variável distensão dos cornos e conteúdo intrauterino de aspecto translúcido e mucoide. Dessa forma, uma vez observadas alterações as quais poderiam indicar de alguma afecção e pelo fato da técnica de OVE não ser indicada nos casos de enfermidades uterinas (Ehrhardt, 2012), alguns animais foram incluídos no grupo $\mathrm{OVH}$ como forma de tratamento e/ou prevenção, conforme recomenda a literatura (Slatter, 2007; Rolim et al., 2010; Copat, 2015).

Algumas doenças uterinas podem não causar sinais clínicos, como HEC, mucometra, hidrometra e hemometra (Verstegen et al., 2008). Assim como a ausência de sinais clínicos, a normalidade nos exames complementares, como hemograma e bioquímico, também podem ser observadas em casos de mucometra, sendo esta muitas vezes diagnosticada acidentalmente durante o procedimento cirúrgico de OVH (Stone, 2007; Gonsales, 2010), conforme ocorreu em seis pacientes deste experimento. Os autores supracitados afirmam ainda que com o auxílio da ultrassonografia, seria possível observar o aumento de volume uterino, embora esse exame não possa ser usado para diagnóstico definitivo.

Acredita-se que a HEC é comumente desenvolvida em cadelas em algum momento de sua vida (Ettinger e Feldman, 2004), sendo a castração indicada como forma de prevenção desta e de outras afecções, e a OVE é uma das técnicas mais utilizadas. Contudo, não foram observadas na literatura evidências de que cadelas acometidas por HEC, submetidas à OVE, sem o prévio conhecimento da instalação do complexo, possam 
vir, a longo prazo, apresentar uma evolução do HEC para mucometra ou piometra, por exemplo. Desta forma seria necessário o acompanhamento das cadelas diagnosticadas com HEC, submetidas à OVE no presente estudo, para elucidação dessas suspeitas.

\section{Conclusão}

Os resultados obtidos neste trabalho permitem concluir que o exame histopatológico é fundamental no diagnóstico de alterações ovarianas e/ou uterinas não detectadas em exames clínico, ultrassonográfico, laboratorial (hemograma e bioquímico), e macroscópico de cadelas submetidas à castração eletiva pelas técnicas de OVH e OVE.

\section{Conflito de Interesse}

Os autores declaram não existir conflito de interesse.

\section{Comitê de Ética}

O manejo e os cuidados dos animais foram realizados de acordo com as diretrizes e recomendações da Comissão de Ética no Uso de Animais (CEUA) da UFRPE, sob o número de licença 003/2018.

\section{Referências}

Aguirra, L.R.V.M.; Pereira, W.L.A.; Marsolla, E.H. Ocorrência e aspectos macro e microscópicos da mucometra em cadelas. Veterinária e Zootecnia, 22(1): 83-88, 2015.

Araujo, E.D.B.; Silva, L.F.M.C.; Oliveira, S.N.; Dalanezi, F.M.; Costa, L.D.; Rodrigues, J.C.; Zahn, F.S.; Prestes, N.C. Fibroma uterino em cadela. Revista Brasileira de Ciência Veterinária, 22(2): 119-123, 2015.

Copat, B.; Chaves, R.O.; Feranti, J.P.S.; Coradini, G., Hartmann, H.F., Corrêa, L.F.D., Oliveira, M.T.; Brun, M.V. Ovariohisterectomia videolaparoscópica em cadela com ovários remanescentes e piometra de cornos uterinos. Acta Scientiae Veterinariae, 43(1): 97, 2015.

Carreira, P.R.; Pires, M.A. Hiperplasia quística do endométrio em cadelas. Revista Portuguesa de Ciências Veterinárias, 100: 5-16, 2005.

Daleck, C.R.; De Nardi, A.B.; Rodaski, S. Neoplasias do sistema reprodutor feminino. In: Neoplasias em cães e gatos. São Paulo: Roca, 2009. p.354-359.

De Bosschere, H.; Ducatelle, R.; Tshamala, M.; Coryn, M. The use of epostane in an attempt to reproduce cystic endometrial hyplerplasia in the bitch. Veterinary Research Comunications, 27(7): 527-538, 2003.

Dow. C. Experimental reproduction of the cystic hyperplasia-pymetra complex in the bitch. Journal of Pathology and Bacteriology, 78(1): 267-278, 1959.

Ehrhardt, E. E. Performing an ovariectomy in dogs and cats. Veterinary Medicine, 107(6): 272, 2012.

Ettinguer, S.J.; Feldman, E.C. Tratado de medicina interna veterinária. $5^{\mathrm{a}} \mathrm{ed}$. Rio de Janeiro: Guanabara Koogan, 2004. p. 16321634.

Fossum, T.W. Cirurgia de pequenos animais. $2^{\mathrm{a}}$ ed. São Paulo: Roca. 2005, 1640p.

Gonsales F.F. Distúrbios da vagina e do útero. In: Nelson, W.R; Couto, G.C. Medicina interna de pequenos animais. Rio de Janeiro: Elsevier, 2010. p.921-922.

Howe, L.M. Surgical methods of contraception and sterilization. Theriogenology, 66(3): 500509, 2006.

Jiang, J.; Tate, Y.; Kobayashi, Y.; Ichikawa, A. Adenomatous hyperplasia of the rete ovarii in a beagle. Journal of Toxicologic Pathology, 17(2): 127-128, 2004.

Jones, T.C.; Hunt, R.D.; King, N.W. Sistema urogenital. In: Patologia veterinária. São Paulo: Manole. 2000. p.1169-1197.

Kennedy, P.C.; Cullen, J.M.; Edwards, J.F.; Goldschmidt, M.H.; Larsen, S.; Munson, L.; Nielsen, S. World Health Organization International Histological Classification of Tumors of Domestic Animals. Histological Classification of Tumors of the Genital System of Domestic Animals. $2^{\text {nd }}$ ed. Washington: Armed Forces Institute of Pathology, American Registry of Pathology, 1998, 79p.

Luna, L.G. Manual of histological staining methods of armed forces institute of pathology. Washington: MacGraw Hill, 1968. 258p.

Maclachlan, N.J.; Kennedy, P.C. Tumors of the genital system. In: Tumors in domestic animals. Iowa: Iowa State Press. 2002. p.54773.

Macphail, C. Surgery of the reproductive and genital systems. Veterinary Clinics of North America: Small Animal Practice, 44(2): 1931, 2014. 
McEntee, K. Reproductive pathology of domestic mammals. San Diego: Academic Press. 1990. 401p.

Monteiro, C.M.R.; Perri, S.H.V.; Carvalho, R.G. Histologia e morfometria em cornos uterinos de cadelas nulíparas e tratadas com contraceptivos. Pesquisa Veterinária Brasileira, 29(10): 847-851, 2009.

Nascimento, E.F.; Santos, R.L. Patologias do útero. In: Patologia da reprodução de animais domésticos. Rio de Janeiro: GuanabaraKoogan, 2011, p.44-61.

Nogales, F.F.; Carvia, R.E.; Donne, C.; Campelo, T.R. Adenomas of the rete ovarii. Human Pathology, 28(12): 1428-1433, 1997.

Okkens, A.C.; Kooistra, H.S.; Nickel, R.F. Comparison of long term side effects of ovariectomy versus ovariohisterectomy in the bitch. Tijdschrift voor Diergeneeskunde, 127(11): 369-372, 2002.

Pimentel, F.G. Curso de estatística experimental. São Paulo: Nobel. 1985. 467p.

Rolim, V.M.; Pinto, T.M.; Lopes, L.M.A. Sonne, L.; Oliveira, E.C.D.; Almeida, P.R.D.; Beck, C.A.C.; Driemeier, D. Disgerminoma bilateral e hyperplasia endometrial cística com piometra em cadela. Acta Scientiae Veterinariae, 38(3): 337-340, 2010.
Santos, F.C.; Corrêa, T.P.; Rahal, S.C.; Crespilho, A.M.; Lopes, M.D.; Mamprim, M.J. Complicações da esterilização cirúrgica caninas e felinas- revisão de literatura. Veterinária e Zootecnia, 16(1): 08-18, 2009. Shariati, R.; Bakhtiari, J.; Khalaj, A.; Naslaji, AN. Comparison between two portal laparoscopy and open surgery for ovariectomy in dogs. Veterinary Research Forum, 5(3): 219-223, 2014.

Slatter. D. Manual de cirurgia de pequenos animais. São Paulo: Manole, 2007.

Stone E.A. Sistema reprodutivo. In: Slatter D. Manual de cirurgia de pequenos animais. Barueri: Manole, 2007. p.1493 - 1499.

Veiga, G.A.L.; Miziara, R.H.; Angrimani, D.S.R.; Papa, P.C.; Cogliati, B.; Vannucchi, C.I. Cystic endometrial hyperplasia-pyometra syndrome in bitches: identification of hemodynamic, inflammatory, and cell proliferation changes. Biology of Reproduction, 96(1): 58-69, 2017.

Verstegen J., Dhaliwal G. Verstegen-Onclin K. Mucometra, cystic endometrial hyperplasia, and pyometra in the bitch: advances in treatment and assessment of future reproductive success. Theriogenology, 70: 364-374, 2008. 